\title{
Editorial Comment: Effect of smoking cessation on sexual function in men aged 30 to 60 years
}

\author{
Carlos Teodósio Da Ros ${ }^{1,2}$, Fernando Nestor Facio Jr. ${ }^{2,3}$ \\ ${ }^{1}$ DR\&G Urologistas Associados, Porto Alegre, RS, Brasil; ${ }^{2}$ Departamento de Andrologia, Sociedade Bra- \\ sileira de Urologia - SBU, Rio de Janeiro, RJ, Brasil; ${ }^{3}$ Departamento de Urologia, Faculdade de Medicina \\ de São José Rio Preto, São José do Rio Preto, SP, Brasil
}

\section{COMMENT}

This study involved 181 relatively young individuals (30-60 years) who were former smokers and had no other risk factor for erectile dysfunction (ED) besides smoking. The participants completed the IIEF during the first consultation, while still under the effects of smoking, and again six months later. As expected, the prevalence of ED was significantly lower after smoking cessation (1).

While much is focused on smoking and its association with cancer and cardiovascular disease, which occur in older individuals, few studies address the effects on young people and adolescents, who can suffer the same adverse effects, including ED. There are approximately one billion smokers in the world and every year eight million people die due to smoking and its adverse effects (2).

The pathophysiological mechanism of endothelial dysfunction results from an inhibition of the nitric oxide cascade, preventing adequate arterial dilation and the blood flow necessary for penile erection. In addition to endothelial dysfunction, smoking is also a risk factor for arteriosclerosis (3-5). Sahin MO et al., the authors of the study commented here, also mention the correlation between ED and cardiovascular disease and smoking as a risk factor for both (1).

In smokers with heart disease, the risk of complete ED is seven times higher than that imposed by any of the risk factors alone (6). In an analysis of more than 31,000 individuals over 50 years of age, the prevalence ED was 33\% and was higher among those who were obese, sedentary, smokers and alcoholics (7). These data were confirmed in several other studies $(8,9)$.

This study offers insight into contemporary indications that smoking is significantly associated with ED and smoking cessation has a beneficial effect on the restoration of erectile function (EF). The literature offers studies showing the association between smoking and hypertension, acute coronary syndrome, angina, atherosclerosis, cerebrovascular diseases and sudden death. Based on this consistency, a fair conclusion may be drawn that male sexual function and smoking have a temporal relation; smoking precedes ED. There is an increased risk of ED with greater exposure to smoking and smoking cessation can lead to the recovery of erectile function but only if lifetime exposure to smoking is limited $(10,11)$.

Based on this study, urologists should counsel smokers with ED to quit smoking, which will result in an improvement in erectile rigidity and tumescence.

\section{CONFLICT OF INTEREST}

None declared. 


\section{REFERENCES}

1. Sahin MO, Sen V, Gunduz G, Ucer O. Effect of smoking cessation on sexual functions in men aged 30 to 60 years Int Braz J Urol. 2020;46:642-8.

2. WHO. Tobacco. Available at. <https://www.who.int/newsroom/fact-sheets/detail/tobacco $>$

3. Ponholzer A, Stopfer J, Bayer G, Susani M, Steinbacher F, Herbst $F$, et al. Is penile atherosclerosis the link between erectile dysfunction and cardiovascular risk? An autopsy study. Int J Impot Res. 2012;24:137-40.

4. Siroky MB, Azadzoi KM. Vasculogenic erectile dysfunction: newer therapeutic strategies. J Urol. 2003;170(2 Pt 2):S249; discussion S29-30.

5. Kendirci M, Trost L, Sikka SC, Hellstrom WJ. The effect of vascular risk factors on penile vascular status in men with erectile dysfunction. J Urol. 2007;178:2516-20; discussion 2520.pub 2007 Oct 15. PubMed PMID: 17937942.

6. Feldman $H A$, Goldstein I, Hatzichristou DG, Krane RJ, McKinlay JB. Impotence and its medical and psychosocial correlates: results of the Massachusetts Male Aging Study. J Urol. 1994;151:54-61.
7. Bacon CG, Mittleman MA, Kawachi I, Giovannucci E, Glasser $D B$, Rimm EB. Sexual function in men older than 50 years of age: results from the health professionals follow-up study. Ann Intern Med. 2003;139:161-8.

8. Allen MS, Walter EE. Erectile Dysfunction: An Umbrella Review of Meta-Analyses of Risk-Factors, Treatment, and Prevalence Outcomes. J Sex Med. 2019;16:531-41.

9. Braun M, Wassmer G, Klotz T, Reifenrath B, Mathers M, Engelmann U. Epidemiology of erectile dysfunction: results of the 'Cologne Male Survey'. Int J Impot Res. 2000;12:305-11.

10. Biebel MG, Burnett AL, Sadeghi-Nejad H. Male Sexual Function and Smoking. Sex Med Rev. 2016;4:366-75.

11. Rosen MP, Greenfield AJ, Walker TG, Grant P, Dubrow J, Bettmann MA, et al. Cigarette smoking: an independent risk factor for atherosclerosis in the hypogastric-cavernous arterial bed of men with arteriogenic impotence. J Urol. 1991;145:759-63.
ARTICLE INFO

Carlos Teodósio Da Ros

https://orcid.org/0000-0002-4768-7239

Int Braz J Urol. 2020; 46: 649-50

\section{Carlos Teodósio Da Ros, MD}

DREG Urologistas Associados

Avenida Lageado, 1212,808

Petrópolis, Porto Alegre - RS, 90460-110, Brasil

Fax: +55 51 98166-8777

E-mail: daroscarlos@yahoo.com

Accepted:

March 31, 2020 